Research Article

\title{
Simultaneous Quantification of Paracetamol and Caffeine in Powder Blends for Tableting by NIR-Chemometry
}

\author{
Dana Maria Muntean, ${ }^{1}$ Cristian Alecu, ${ }^{2}$ and Ioan Tomuta ${ }^{1}$ \\ ${ }^{1}$ Department of Pharmaceutical Technology and Biopharmacy, University of Medicine and Pharmacy Iuliu Hatieganu Cluj-Napoca, \\ 41 V. Babes, 400012 Cluj-Napoca, Romania \\ ${ }^{2}$ S.C. Laropharm S.R.L., 145A Alexandriei, 70000 Bragadiru, Romania
}

Correspondence should be addressed to Ioan Tomuta; tomutaioan@umfcluj.ro

Received 1 February 2017; Accepted 6 April 2017; Published 28 May 2017

Academic Editor: Rizwan Hasan Khan

Copyright (C) 2017 Dana Maria Muntean et al. This is an open access article distributed under the Creative Commons Attribution License, which permits unrestricted use, distribution, and reproduction in any medium, provided the original work is properly cited.

Near-infrared spectroscopy (NIRS) is a technique widely used for rapid and nondestructive analysis of solid samples. A method for simultaneous analysis of the two components of paracetamol and caffeine from powder blends has been developed by using chemometry with near-infrared spectroscopy (NIRS). The method development was performed on samples containing 80,90 , 100,110 , and $120 \%$ active pharmaceutical ingredients, and near-infrared spectroscopy (NIRS) spectra of prepared powder blends were recorded and analyzed in order to develop models for the prediction of drug content. Many calibration models were applied in order to perform quantitative determination of drug content in powder, and choosing the appropriate number of factors (principal components) proved to be of highly importance for a PLS chemometric calibration. Once the methods were developed, they were validated in terms of trueness, precision, and accuracy. The results obtained by NIRS methods were compared with those obtained by HPLC reference method, and no significant differences were found. Therefore, the NIR chemometry methods were proved to be a suitable tool for predicting chemical properties of powder blends and for simultaneous determination of active pharmaceutical ingredients.

\section{Introduction}

Near-infrared spectroscopy (NIRS) has been proved to remain a powerful analytical tool for analyzing a vast variety of samples from petrochemical, food, agricultural, and pharmaceutical industries [1]. During the last decade, it has experienced a significant increase for quantitative pharmaceutical tests. The quantitative analytical strategies concerning pharmaceuticals, such as HPLC, GC, and UV spectrophotometry, usually require dissolving the samples, separating them, and determining their ingredients, being destructive. Compared to traditional analytical methods, near-infrared spectroscopy (NIRS) has been proved to be a more versatile and fastgrowing analytical tool not only in the pharmaceutical sciences but also in the industry. The main advantages of this technique are that it is noninvasive and nondestructive, has no sample preparation required, large number of molecules which could be quantified, and is very fast due to the high frequency of spectrum acquisition [2].
In recent years, NIRS methods are starting to become popular in the pharmaceutical area. NIRS needs chemometric analysis of data in order to be used as quantitative technique. Chemometric methods such as three linear regression modeling methods, principal component regression (PCR), partial least squares (PLS) [3], or one nonlinear regression model based on artificial neural networks (ANN) $[4,5]$ are used [6, 7]. For the determination of the concentrations' chemical compounds in pharmaceutical field using near-infrared spectroscopy, PCR and PLS models are intensively used [6-8].

In order to increase patient compliance, the combination of two or more active compounds in the same commercial preparation may be used. The manufacturing process of tablets containing two or more active compounds, such us fixed-dose combination tablets, involves the following unit operations: dispersing, granulation, mixing, tableting, and coating. Each of the unit operations may have huge influence on the quality of the final product. For example, powder 
mixing is an essential unit operation for manufacturing fixed-dose combination, because inadequate mixing process conducts to poor quality of the final product due to low blend uniformity that is critical to ensure compliant content uniformity per united dose [9]. An important goal for NIRS analysis in pharmaceutical industry is a direct evaluation of powder blends during the mixing process as on-line, in-line, or at-line testing $[2,10]$.

Paracetamol ( $\mathrm{N}$-acetyl-p-aminophenol, acetaminophen) is a long-established substance, being one of the most extensively employed drugs in the world. For patients with sensitivity to aspirin, it is a noncarcinogenic and effective substitute and it is accepted to be a suitable drug for the relief of pain and fever in adults and children [11]. Due to its intensive use for therapeutic purposes, the quick methods for its determination during quality control are of great importance. In last years, many methods for paracetamol determination have been reported, such as chromatographic, spectrofluorimetric, chemiluminiscent, spectrophotometric, and electrochemical techniques [12].

Caffeine (3,7-dihydro-1,3,7-trimethyl-1H-purine-2,6dione) is an alkaloid $\mathrm{N}$-methyl derivative of xanthine that is broadly distributed in natural products, commonly used in beverages. Its consumption has many physiological effects, such as gastric acid secretion, diuresis, and stimulation of the central nervous system [13]. It is used in combination with nonsteroidal anti-inflamatory drugs in analgesic formulations or in combination with ergotamine in migraine treatment. The special literature reveals various methods for caffeine analyzing, including spectrophotometric [14, 15] and chromatographic [16] ones. These methods are generally expensive, time consuming, and complicated.

HPLC methods are widely used for powder blend uniformity evaluation, due to good selectivity, specificity, and linear range. However, this technique requires sample preparation and chromatographic separation of the analytes, so it takes hours and therefore can be done only offline. Monitoring the blend uniformity in the mixing steps of tablet manufacturing is considered to be an important goal for PAT concept and can be done only by a direct analysis. Once calibrated and validated, NIRS methods seem to be the best analytical options, due to short analysis time and low cost per analysis in contrast with HPLC analysis methods.

In this paper, we explored the applications of chemometry on near-infrared spectroscopy (NIRS) for the quantitative analysis of paracetamol and caffeine, in powder blend for tableting, predicting their concentrations simultaneously, without any processing of the sample.

\section{Materials and Method}

2.1. Materials. All the raw material powders including paracetamol (Novacyl, France) and anhydrous caffeine (BASF, Germany) as active compounds and lactose (Meggle, Germany), cornstarch (Roquette, France), colloidal silicon dioxide-Aerosil (Rohm Pharma Polymers, Germany), polyvinyl-pyrrolidone (BASF, Germany), talcum (IMERYS Luzenac, France), and magnesium stearate (Union Derivan S.A, Spain) as excipients were pharmaceutical grade.
TABLE 1: Composition of calibration and validation samples.

\begin{tabular}{lccccc}
\hline Concentration level & 1 & 2 & 3 & 4 & 5 \\
& $80 \%$ & $90 \%$ & $100 \%$ & $110 \%$ & $120 \%$ \\
\hline Paracetamol & 38.71 & 43.55 & 48.39 & 53.23 & 58.06 \\
Caffeine & 3.84 & 4.35 & 4.84 & 5.32 & 5.81 \\
\hline \multicolumn{5}{c}{ Tablets composition } & (mg/tablet) \\
Paracetamol & 240.0 & 270.0 & 300.0 & 330.0 & 360.0 \\
Caffeine & 24.0 & 27.0 & 30.0 & 33.0 & 36.0 \\
Lactose & 146.0 & 113.0 & 80.0 & 47.0 & 14.0 \\
Cornstarch & & & & & \\
Colloidal silicon dioxide & & & & & \\
PVP & 210.0 & 210.0 & 210.0 & 210.0 & 210.0
\end{tabular}

Talcum

Magnesium stearate

\begin{tabular}{llllll}
\hline Tablet weight & 620.0 & 620.0 & 620.0 & 620.0 & 620.0 \\
\hline
\end{tabular}

Calibration samples: levels 1, 2, 3, 4, and 5. Validation samples: levels 2, 3, and 4 .

2.2. Sample Preparation. Pharmaceutical industries frequently use wet granulation in order to convert fine cohesive powders into dense and round granules. The granules are produced by vigorous mixing of a wet-powdered mixture composed of active compounds, some excipients, and binder. The overall purpose of this operation is to obtain a final product with improved characteristics, such as better flowability and compressibility. Other benefits were obtained using wet granulation; the distribution of the drug in the final product, as well as the dissolution properties of tablets may be improved.

For calibration and validation purpose, powder blends for tablets were prepared as presented in Table 1.

Paracetamol, caffeine, lactose, some cornstarch, and PVP (which was previously dissolved in $4.8 \mathrm{ml}$ distilled water) were mixed. The wet mixture was passed through a sieve. It was left to dry at room temperature until the next day; when it was weighted, the amount of remaining excipients to be added was adjusted according to the weighted mixture. The powder blend was passed through a $800 \mu \mathrm{m}$ sieve.

The mixture composition was designed for a tablet weight of approximately $620 \mathrm{mg}$ and a usual amount of active ingredients of $300 \mathrm{mg}$ paracetamol (48.38\%, w/w) and $30 \mathrm{mg}$ caffeine $(4.84 \%, w / w)$. This formulation will be further considered as $100 \%$ active content formulation.

2.3. Experimental Design for Model Development. A calibration set was built using a full factorial experimental design with two factors and two levels, using Modde 11.0 Software (Umetrics, Sweden) to build and analyze the experimental plans. Each sample in the calibration set contained two components (paracetamol, caffeine); each component was taken at five concentration levels (80, 90, 100, 110, and $120 \%$ reported to the theoretical amount). The composition of calibration set samples is presented in a full factorial matrix of experimental plan, in Table 2. 
TABle 2: Experimental design matrix for calibration set.

\begin{tabular}{lccccccc}
\hline $\begin{array}{l}\text { Exp } \\
\text { name }\end{array}$ & $\begin{array}{c}\text { Run } \\
\text { order }\end{array}$ & $\mathrm{X}_{1}$ & $\mathrm{X}_{2}$ & $\begin{array}{c}\text { Exp } \\
\text { name }\end{array}$ & $\begin{array}{c}\text { Run } \\
\text { order }\end{array}$ & $\mathrm{X}_{1}$ & $\mathrm{X}_{2}$ \\
\hline $\mathrm{N} 1$ & 9 & 38.71 & 3.87 & $\mathrm{~N} 15$ & 13 & 58.06 & 4.84 \\
$\mathrm{~N} 2$ & 26 & 43.55 & 3.87 & $\mathrm{~N} 16$ & 25 & 38.71 & 5.32 \\
N3 & 16 & 48.39 & 3.87 & $\mathrm{~N} 17$ & 24 & 43.55 & 5.32 \\
$\mathrm{~N} 4$ & 21 & 53.23 & 3.87 & $\mathrm{~N} 18$ & 17 & 48.39 & 5.32 \\
N5 & 11 & 58.06 & 3.87 & $\mathrm{~N} 19^{*}$ & $\mathbf{4}$ & $\mathbf{5 3 . 2 3}$ & $\mathbf{5 . 3 2}$ \\
N6 & 15 & 38.71 & 4.35 & $\mathrm{~N} 20$ & 7 & 58.06 & 5.32 \\
N7* & $\mathbf{8}$ & $\mathbf{4 3 . 5 5}$ & $\mathbf{4 . 3 5}$ & $\mathrm{N} 21$ & 28 & 38.71 & 5.81 \\
N8 & 6 & 48.39 & 4.35 & $\mathrm{~N} 22$ & 1 & 43.55 & 5.81 \\
N9 & 27 & 53.23 & 4.35 & $\mathrm{~N} 23$ & 3 & 48.39 & 5.81 \\
N10 & 23 & 58.06 & 4.35 & $\mathrm{~N} 24$ & 18 & 53.23 & 5.81 \\
N11 & 14 & 38.71 & 4.84 & $\mathrm{~N} 25$ & 22 & 58.06 & 5.81 \\
N12 & 20 & 43.55 & 4.84 & N26 & 12 & 48.39 & 4.84 \\
N13* & $\mathbf{2}$ & $\mathbf{4 8 . 3 9}$ & $\mathbf{4 . 8 4}$ & $\mathrm{N} 27$ & 19 & 48.39 & 4.84 \\
N14 & 10 & 53.23 & 4.84 & N28 & 5 & 58.06 & 4.84 \\
\hline
\end{tabular}

$\mathrm{X}_{1}$-paracetamol concentration ( $\mathrm{mg} /$ tablet); $\mathrm{X}_{2}$-caffeine concentration (mg/tablet). ${ }^{*}$ Validation samples.

2.4. Recording of NIR Spectra. Near-infrared spectra of powder blends were recorded using a Fourier-transform NIRS analyzer (Antaris II, ThermoElectron Scientific, USA) in reflectance sampling configuration, equipped with an indium gallium arsenide (InGaAs) detector. Since the powder samples are not homogeneous, the device is equipped with a system for the rotation of samples during the measurements, so that obtained spectrum is representative for the sample and ensures the reproducibility of measurements. Each reflectance spectrum was recorded using OMNIC software (Termo Scientific, USA) by integrating 32 scans, over the range of 11000 to $4000 \mathrm{~cm}^{-1}$, with a resolution of $8 \mathrm{~cm}^{-1}$.

2.5. HPLC as Reference Method. After the collection of all NIR spectra from each individual powder blend, highperformance liquid chromatography (HPLC) analysis was performed for reference. Weighted powder samples were dissolved in methanol, in a $25 \mathrm{ml}$ volumetric flask. The flask was vibrated in an ultrasonic bath (Transsonic T700, Germany) until complete dissolution. $5 \mathrm{ml}$ were transferred to a centrifuging tube and were centrifuged (Sigma 2-16, Sartorius, Germany) for $5 \mathrm{~min}$ at $5000 \mathrm{rpm}$. One milliliter of the resulting supernatant was pipetted into a $10 \mathrm{ml}$ volumetric flask and diluted to volume with water-acetonitrile $(75: 25, \mathrm{v} / \mathrm{v})$

Separately, $10 \mathrm{mg}$ of paracetamol and $10 \mathrm{mg}$ of caffeine were accurately weighted (using $0,01 \mathrm{mg}$ analytical balance) into a $10 \mathrm{ml}$ volumetric flask, and the same operation as described above was carried out to prepare the standard solution for calibration curve.

The samples were then analyzed by HPLC with UV detection. The HPLC system was a 1100 series model (Agilent Technologies, USA) consisted in a binary pump, an autosampler, a column thermostat, and a UV detector. Separation was carried out on a Gemini C18 $(100 \times 3.00 \mathrm{~mm} ; 3 \mu \mathrm{m})$ column, with a mobile phase consisting of acetate buffer-acetonitrile

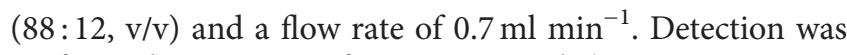
performed at $245 \mathrm{~nm}$ for paracetamol (retention time at $1.9 \mathrm{~min}$ ) and $275 \mathrm{~nm}$ for caffeine (retention time at $2.9 \mathrm{~min}$ ). The calibration curves used for paracetamol and caffeine determination were in the range of $40-200 \mu \mathrm{g} \mathrm{ml}^{-1}$ and $4-$ $20 \mu \mathrm{g} \mathrm{ml}^{-1}$, respectively.

2.6. Data Processing. NIR spectra recorded for multivariate calibration models were previously processed using several established methods: first derivative, second derivative, standard normal variate (SNV), multiplicative scattering correction (MSC), straight line subtraction (SLS), minimum maxim normalization (MMN), in order to construct the calibration models. The partial least square (PLS) regression was conducted using multivariate analysis Opus Quant software (Bruker Optics, Germany).

This software allows validation of the chemometric multivariate calibration by the "full cross-validation." According to this procedure, iterative calibrations were performed by removing in turn each standard from the training set and then predicting the excluded sample with that calibration [17].

2.7. Method Validation. Once a calibration is developed and favourable predictions are expected, they must be validated to be accepted for routine use. Independent sets of samples are needed for external validation. There are several validation parameters that must be determined in order to be consistent with the recommendations of the International Conference of Harmonization (ICH) and with other guidelines: accuracy, precision (repeatability and intermediate precision), linearity, and range of application. The validation was performed according to the strategy proposed by Hubert et al., $[18,19]$ and adapted by the authors for NIR methods [10, 22, 23].

Validation of NIR methods for both paracetamol and caffeine assays was performed considering $90 \%, 100 \%$, and $110 \%$ active compound content (formulations N7, N13, and N23). Four replicates were prepared for each formulation, in three different days, resulting a 36-sample validation set. In order to see which model fits the best, linearity and accuracy profiles were computed and compared, considering $\mathrm{ICH}$ Q2 guideline requirements.

\section{Results and Discussion}

Development of chemometric multivariate calibration models means to calculate the calibration parameters of the obtained data after the analysis of the NIR spectral calibration set. To do this, various methods of pretreatment of spectra in combination with selecting different spectral regions and different methods of regression analysis may be used. The entire spectrum and selecting certain spectral regions containing strong absorption bands in combination with different pretreatment methods of single spectra as standard normal variate (SNV), first derivative (FD), multiplicative scattering correction (MSC), straight line subtraction (SLS), minimum maximum normalization (MMN), or combined $(\mathrm{FD}+\mathrm{SNV}, \mathrm{FD}+\mathrm{MSC}$, and FD + SLS) were tested for building a calibration model. Once the calibration model has been 


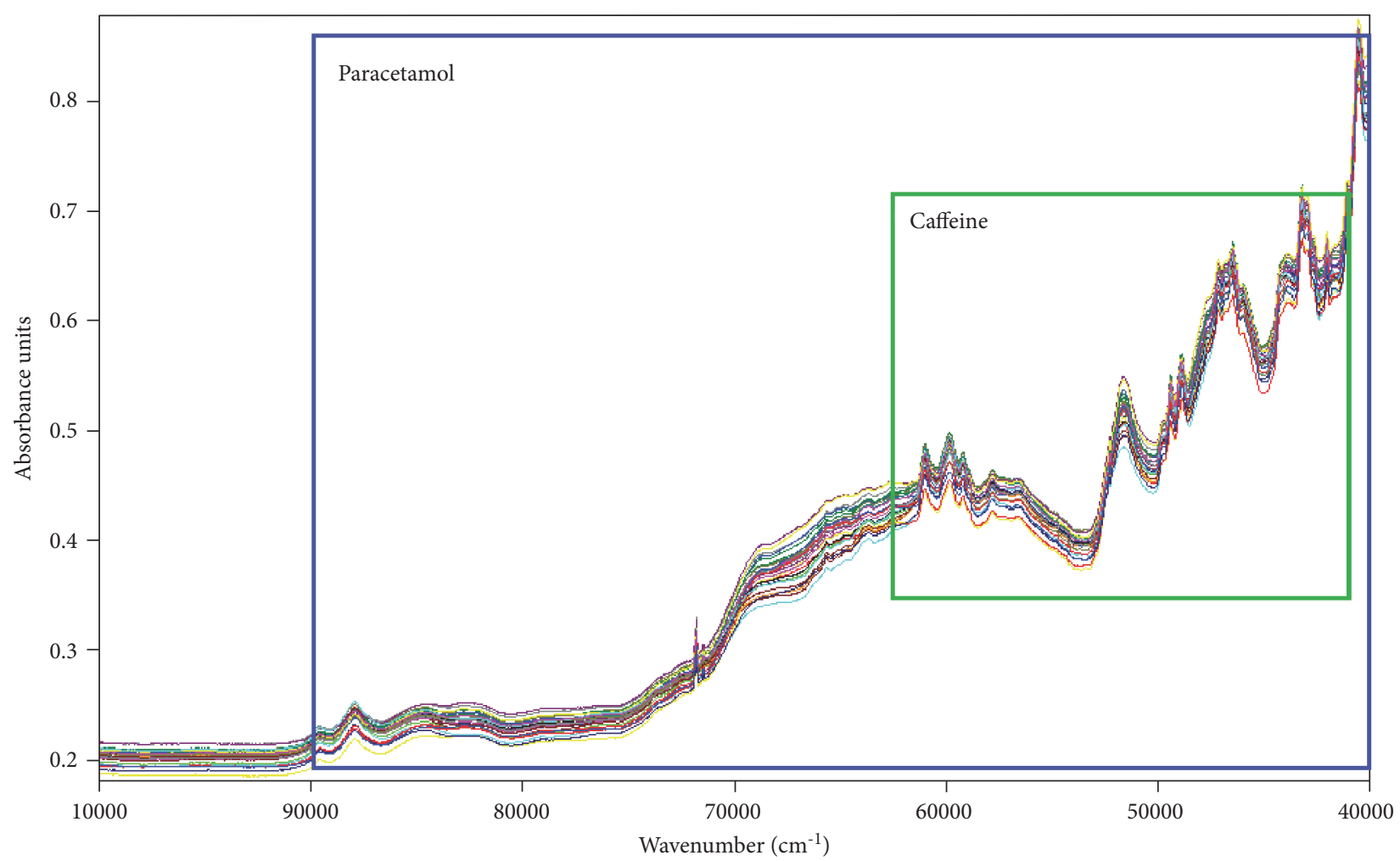

FIGURE 1: Reflectance spectrum of powder mixture recorded at a resolution of $8 \mathrm{~cm}^{-1}$ for a calibration set. The highlighted blue area defines the spectral ranges selected for the paracetamol, and the green area defines the spectral ranges selected for the caffeine.

developed, the capacity of prediction was tested on the test samples used during development.

3.1. Spectral Investigation. The NIR reflectance spectrum of the calibration set is presented in Figure 1. The calibration model for powder blends was built after recording three spectra of each formulation. Overall, 84 spectra were recorded and analyzed. NIR spectra of powder blends contain both chemical and physical information, so robust calibration models must be developed. Therefore, preprocessing methods and wavelength selection ranges should be carefully chosen to extract the chemical information that is mainly correlated with the API concentration [9]. In the spectral region with wavelengths less than $4000 \mathrm{~cm}^{-1}$, the amount of radiation reaching the detector is reduced, background noise is high, and therefore it was eliminated from the analysis. Eliminating these spectral regions was carried out by other researchers too, who found similar results $[20,21]$. Thus, model development for paracetamol assay was done using the spectral regions $9000-4000 \mathrm{~cm}^{-1}$. In the case of caffeine, the spectral regions selected for model development and future analysis were $6200-4100 \mathrm{~cm}^{-1}$.

3.2. Model Development and Validation for Active Compounds. Many calibration models were applied in order to perform quantitative determination of drug content in powder. Choosing the appropriate number of factors (principal components) is highly important for a PLS chemometric calibration. The number of factors for the experimental data obtained in calibration has to be chosen so that to avoid "over fitting." There were different methods proposed in order to select the optimum number of factors [22, 23]. Root mean square error of prediction (RMSEP) was used as a diagnostic test to assess the predictive ability of the model. Evaluation of prediction models was also made by plotting the known concentrations depending on the estimated concentrations. A good correlation coefficient $R^{2}$ between real and estimated concentrations was obtained for the studied components at optimized models, indicating good predictive ability of the models. The RMSEP and $R^{2}$ values obtained for optimized models for paracetamol are presented in Table 3 and for caffeine in Table 4.

In case that PLS algorithms are used for the development of methods, it is known that the method of spectral data pretreatment and the number of factors (components) are critical parameters. Selecting the optimal number of factors was performed using the criterion of Haaland and Thomas [24-26].

As presented in Table 3, the preprocessing based methods resulted in better prediction ability models. No preprocessing method showed high values for RMSECV, in the comparison of the preprocessing methods. For paracetamol, the models based on spectral range $9000-4000 \mathrm{~cm}^{-1}$ showed good ability of prediction when using 3-6 PLS factors. The authors chose the model $d$ (SNV) showing low RMSECV and good $R^{2}$. For caffeine, the selected spectral range was $6200-4100 \mathrm{~cm}^{-1}$, and the lowest RMSEP and best $R^{2}$ were obtained for the model c (SLS) as can be seen in Table 3 . 
TABLE 3: Statistical parameters and number of PLS factors for different models proposed for paracetamol and caffeine assays in powder blends.

\begin{tabular}{|c|c|c|c|c|c|c|c|c|c|c|c|}
\hline \multicolumn{12}{|c|}{ Paracetamol } \\
\hline Model & $\mathrm{a}$ & $\mathrm{b}$ & $\mathrm{c}$ & $\mathbf{d}^{*}$ & $\mathrm{e}$ & $\mathrm{f}$ & $\mathrm{g}$ & $\mathrm{h}$ & i & j & $\mathrm{k}$ \\
\hline Pretreatment & None & $\mathrm{COE}$ & SLS & SNV & $\mathrm{mMN}$ & MSC & FD & $\mathrm{SD}$ & $\mathrm{FS}+\mathrm{SLS}$ & $\mathrm{FD}+\mathrm{SVN}$ & $\mathrm{FD}+\mathrm{MSC}$ \\
\hline $\begin{array}{l}\text { Spectral range } \\
\text { selected }\left(\mathrm{cm}^{-1}\right)\end{array}$ & & & & & & $9000-4000$ & & & & & \\
\hline Number of PLS factors & 2 & 5 & 6 & 5 & 5 & 5 & 5 & 5 & 5 & 3 & 3 \\
\hline$R^{2}$ & 0.978 & 0.997 & 0.997 & 0.997 & 0.998 & 0.998 & 0.997 & 0.997 & 0.997 & 0.998 & 0.997 \\
\hline RMSECV (\%) & 0.966 & 0.334 & 0.311 & 0.274 & 0.261 & 0.259 & 0.328 & 0.363 & 0.326 & 0.302 & 0.337 \\
\hline Bias (\%) & -0.0075 & 0.00088 & 0.00055 & 0.00044 & -0.0029 & 0.00047 & -0.0093 & 0.0010 & -0.0039 & 0.0098 & 0.0048 \\
\hline \multicolumn{12}{|c|}{ Caffeine } \\
\hline Model & $\mathrm{a}$ & $\mathrm{b}$ & $\mathrm{c}^{*}$ & $\mathrm{~d}$ & $\mathrm{e}$ & $\mathrm{f}$ & $\mathrm{g}$ & $\mathrm{h}$ & $\mathbf{i}$ & $\mathrm{J}$ & $\mathrm{k}$ \\
\hline Pretreatment & None & $\mathrm{COE}$ & SLS & SNV & $\mathrm{mMN}$ & MSC & FD & SD & FS+SLS & $\mathrm{FD}+\mathrm{SVN}$ & $\mathrm{FD}+\mathrm{MSC}$ \\
\hline $\begin{array}{l}\text { Spectral range } \\
\text { selected }\left(\mathrm{cm}^{-1}\right)\end{array}$ & & & & & & $6200-4100$ & & & & & \\
\hline Number of PLS factors & 14 & 14 & 12 & 11 & 11 & 11 & 10 & 8 & 11 & 9 & 9 \\
\hline$R^{2}$ & 0.958 & 0.96 & 0.962 & 0.958 & 0.941 & 0.945 & 0.946 & 0.881 & 0.952 & 0.939 & 0.940 \\
\hline RMSEP & 0.137 & 0.132 & 0.131 & 0.137 & 0.163 & 0.156 & 0.155 & 0.23 & 0.146 & 0.165 & 0.164 \\
\hline Bias (\%) & -0.0019 & -0.0020 & -0.0085 & -0.0036 & -0.0061 & -0.0054 & -0.0046 & -0.0029 & -0.0029 & -0.00407 & -0.0045 \\
\hline
\end{tabular}

*Selected model for validation of the methods.

TABLE 4: Validation results of NIR method for paracetamol and caffeine assays in powder blends.

\begin{tabular}{|c|c|c|c|c|c|c|c|}
\hline \multirow[b]{2}{*}{$\begin{array}{l}\text { Concentration level } \\
\text { (paracetamol) }\end{array}$} & \multirow[b]{2}{*}{$\begin{array}{l}\text { Mean paracetamol } \\
\text { content (mg/tablet) }\end{array}$} & \multicolumn{2}{|c|}{ Trueness } & \multicolumn{2}{|r|}{ Precision } & \multicolumn{2}{|c|}{ Accuracy } \\
\hline & & $\begin{array}{l}\text { Relative } \\
\text { bias (\%) }\end{array}$ & $\begin{array}{c}\text { Recovery } \\
(\%)\end{array}$ & $\begin{array}{l}\text { Repeatability } \\
\text { (RSD \%) }\end{array}$ & $\begin{array}{c}\text { Intermediate precision } \\
(\mathrm{RSD} \%)\end{array}$ & $\begin{array}{l}\text { Relative } \\
\text { tolerance } \\
\text { limits (\%) }\end{array}$ & $\begin{array}{l}\text { Tolerance limits } \\
\text { (mg/tablet) }\end{array}$ \\
\hline \multicolumn{8}{|c|}{ Paracetamol } \\
\hline 43.55 & 43.90 & 0.805 & 100.8 & 0.384 & 0.393 & {$[-0.11,1.72]$} & {$[43.50,44.30]$} \\
\hline 48.39 & 48.44 & 0.101 & 100.1 & 0.188 & 0.185 & {$[-0.32,0.52]$} & {$[48.64,48.64]$} \\
\hline 53.23 & 53.07 & -0.307 & 99.69 & 0.187 & 0.163 & {$[-0.68,0.06]$} & {$[53.26,53.26]$} \\
\hline \multicolumn{8}{|c|}{ Caffeine } \\
\hline 4.355 & 4.437 & 1.880 & 101.88 & 1.34 & 3.19 & {$[-6.26,9.92]$} & {$[4.08,4.82]$} \\
\hline 4.839 & 4.808 & -0.659 & 99.34 & 2.49 & 2.79 & {$[-7.53,6.21]$} & {$[4.48,5.14]$} \\
\hline 5.323 & 5.137 & -1.592 & 98.41 & 1.83 & 1.63 & {$[-5.28,2.01]$} & {$[4.95,5.33]$} \\
\hline
\end{tabular}

Validation of NIR methods for both paracetamol and caffeine assays was performed considering 90\%, 100\%, and $110 \%$ active compound content (formulations N7, N13, and N23). The linear profile, as seen in Figure 2, representing measured concentration versus theoretical concentration of paracetamol and caffeine, respectively, showed a good correlation coefficient and slope, confirming the linearity for the proposed model.

The $\beta$-expectation tolerance limits are included within the $\pm 5 \%$ acceptance limits. Based on the data presented in Table 3 and Figure 3, we concluded that model d is best fitted for paracetamol and model $c$ is best fitted for caffeine assay, in powder blends for tableting. Based on ICH Q2 and EMA guidelines, we validated the following characteristics: linearity, trueness, precision, and accuracy, as shown in Table 4.

The precision of the methods was evaluated by calculating two parameters: repeatability and intermediate precision at three concentration levels. Both parameters had satisfactory values for all concentration levels. The best recovery was obtained for the $100 \%$ paracetamol content, while best intermediate precision was obtained for the $110 \%$ paracetamol content. As for caffeine, the best results in terms of precision were obtained for the $90 \%$ active compound. In terms of accuracy, the $\beta$-expectation tolerance limits are fully included within the $\pm 5 \%$ acceptance limits for both substances, so it can be concluded that the proposed models will provide results with adequate accuracy for both paracetamol and caffeine assays, whatever the active content of the powder blends is within the concentration limits studied.

According to data presented in Figures 2, 3, and Table 4, the NIR-chemometric methods using model $\mathrm{d}$ for paracetamol and model $c$ for caffeine have satisfactory accuracy and linearity profile. It can be concluded that the 


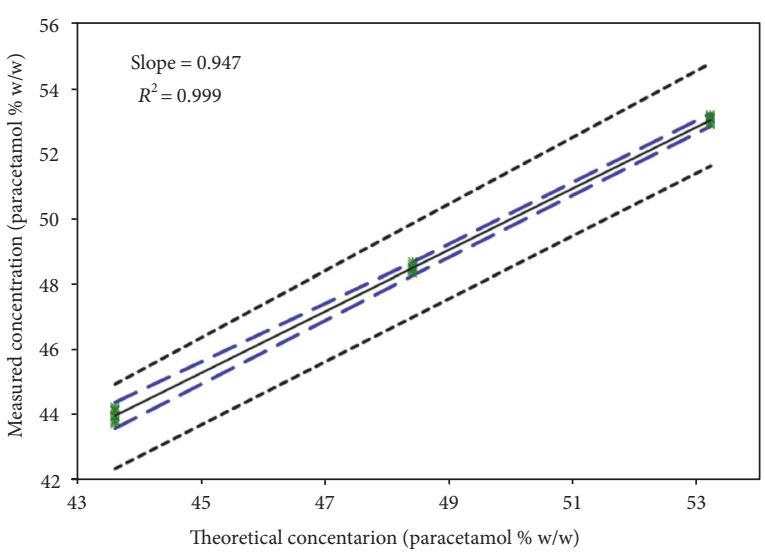

(a)

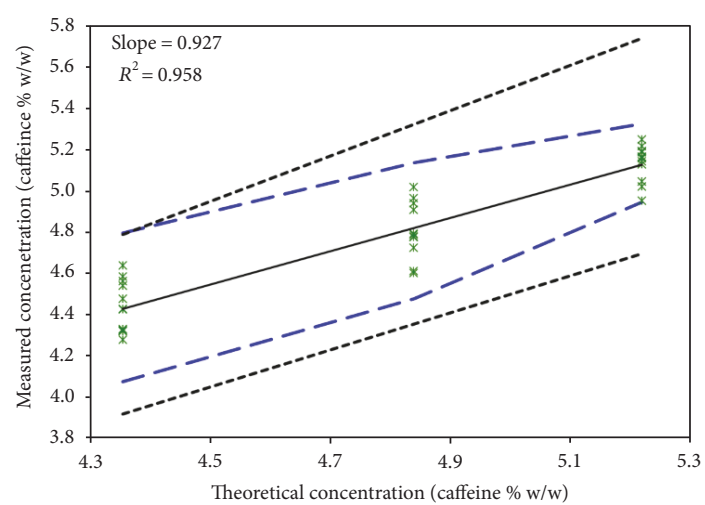

(b)

FIGURE 2: Linearity profile of the NIR—chemometic methods for paracetamol and caffeine determination. (a) paracetamol; (b) caffeine.

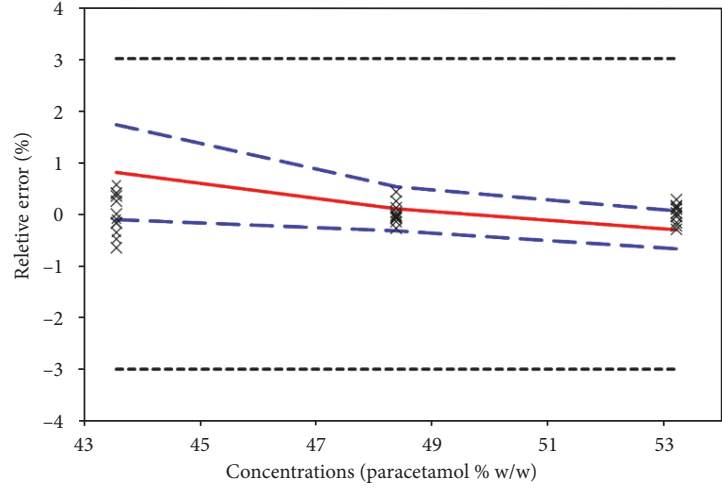

(a)

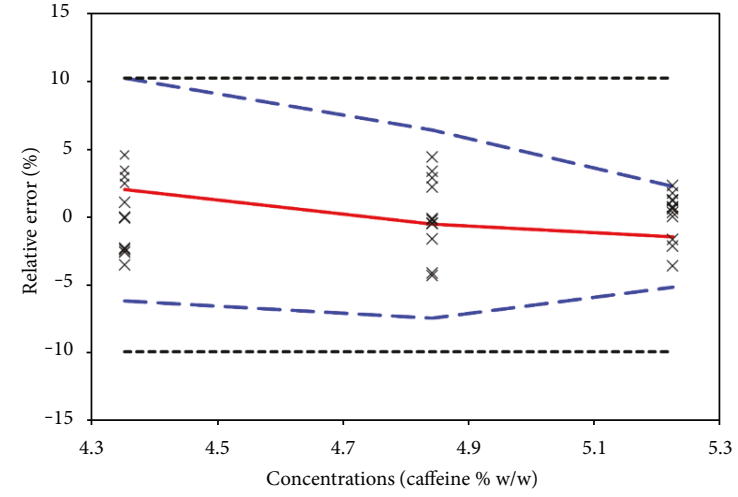

(b)

FIgURE 3: Accuracy profile of the NIR—chemometic methods for paracetamol and caffeine determination. (a) paracetamol; (b) caffeine.

TABLE 5: Paracetamol and caffeine determination by NIR-validated method and HPLC reference method.

\begin{tabular}{lcccc}
\hline API & \multicolumn{2}{c}{ Paracetamol } & \multicolumn{2}{c}{ Caffeine } \\
Method & NIR & HPLC & NIR & HPLC \\
\hline Theoretic concentration (mg) & 48.39 & 48.39 & 4.839 & 4.839 \\
Found (mg) & 48.43 & 48.82 & 4.810 & 4.674 \\
Recovery (\%) & 100.08 & 100.89 & 99.40 & 96.59 \\
CV & 0.17 & 1.19 & 2.15 & 4.53 \\
$t$ value & \multicolumn{2}{c}{1.186} & 1.865 \\
$p$ value & 0.236 & 0.0915 \\
\hline
\end{tabular}

proposed NIR-chemometric method is suitable for paracetamol and caffeine assays in powder blends within the concentration range of $43.55-53.23 \%$ for the paracetamol and 4.35-5.32 for the caffeine.

3.3. Application of the Method. Once the NIR methods for the determination of paracetamol and caffeine were validated, they were applied for the active content determination in six control powder blends containing $48.39 \%$ w/w paracetamol and $4.84 \% \mathrm{w} / \mathrm{w}$ caffeine. The reference HPLC method has been also used for the active content assay in the same samples. The results obtained by the NIR method were compared with the values obtained by the reference HPLC method, in terms of the active content recovery, as shown in Table 5.

As can be seen, the recovery was quite similar for both methods. Also, Student's $t$-test was used to compare the two methods. The results did not show any statistical difference $(p>0.05)$ between the results predicted by NIR and HPLC which are used as the reference method.

\section{Conclusions}

The use of chemometry on near-infrared spectroscopy (NIRS) was explored for nondestructive quantitative analysis of two components in powder blends. The two components were determined simultaneously using pretreated spectra together with chemometry and PLS multivariate calibration. The models were validated in terms of trueness, precision, and accuracy, for an active content of 90,100 , and $110 \%$ for paracetamol and caffeine. Good statistical indicators were obtained. Furthermore, it was proved that the proposed 
methods are suitable for active pharmaceutical ingredient determination, as the results obtained are similar with those obtained by HPLC, which are used as the reference method.

Considering the results presented in this work, the NIR chemometry methods proved to be a suitable tool for predicting the chemical concentration of powder blends during preparation of fixed-dose combination tablets with paracetamol and caffeine. These methods can be used for on-line, inline, or at-line monitoring of the blend uniformity in the mixing steps of the manufacturing process of tablets, with considerable saving in time and money in comparison with HPLC analysis.

\section{Conflicts of Interest}

The authors declare that there is no conflict of interest regarding the publication of this paper.

\section{Acknowledgments}

This work was supported by a grant of the Romanian National Authority for Scientific Research and Innovation, CNCS-UEFISCDI, Project no. PN-III-P2-2.1-BG-2016-0201.

\section{References}

[1] Y. Dou, Y. Sun, Y. Ren, P. Ju, and Y. Ren, "Simultaneous nondestructive determination of two component of combined paracetamol and amantadine hydrochloride in tablets and powder by NIR spectroscopy and artificial neural networks," Journal of Pharmaceutical and Biomedical Analysis, vol. 37, no. 3, pp. 543-549, 2005.

[2] M. Jamrogiewicz, "Application of the near-infrared spectroscopy in the pharmaceutical technology," Journal of Pharmaceutical and Biomedical Analysis, vol. 66, no. 1, pp. 1-10, 2012.

[3] R. G. Brereton, "Introduction to multivariate calibration in analytical chemistry," Analyst, vol. 125, no. 11, pp. 21252154, 2000.

[4] M. Blanco, J. Coello, H. Iturriaga, S. Maspoch, and M. Porcel, "Simultaneous enzymatic spectrophotometric determination of ethanol and methanol by use of artificial neural networks for calibration," Analytica Chimica Acta, vol. 398, no. 1, pp. 83-92, 1999.

[5] A. Safavi, H. Abdollahi, and M. R. Hormezi Nezhad, "Artificial neural networks for simultaneous spectrophotometric differential kinetic determination of $\mathrm{Co}(\mathrm{II})$ and V(IV)," Talanta, vol. 59, no. 3, pp. 515-523, 2003.

[6] T. Wu, H. Chen, Z. Lin, and C. Tan, "Identification and quantitation of melamine in milk by near-infrared spectroscopy and chemometrics," Journal of Spectroscopy, vol. 2016, Article ID 6184987, 8 pages, 2016.

[7] L. Xu, S. M. Yan, C. B. Cai et al., "Nonlinear multivariate calibration of shelf life of preserved eggs (Pidan) by near infrared spectroscopy: stacked least squares support vector machine with ensemble preprocessing," Journal of Spectroscopy, vol. 2013, Article ID 797302, 7 pages, 2013.

[8] J. Irudayaraj and J. Tewari, "Simultaneous monitoring of organic acids and sugars in fresh and processed apple juice by Fourier transform infrared-attenuated total reflection spectroscopy," Applied Spectroscopy, vol. 57, no. 12, pp. 1599-1604, 2003.
[9] L. Martínez, A. Peinado, and L. Liesum, "In-line quantification of two active ingredients in a batch blending process by nearinfrared spectroscopy: influence of physical presentation of the sample," International Journal of Pharmaceutics, vol. 451, no. 1-2, pp. 67-75, 2013.

[10] C. Sîrbu, I. Tomuta, L. Vonica, M. Achim, L. L. Rus, and E. Dinte, "Quantitative characterization of powder blends for tablets with indapamide by near-infrared spectroscopy and chemometry," Farmácia, vol. 62, no. 1, pp. 48-57, 2014.

[11] R. N. Goyal, V. K. Gupta, M. Oyama, and N. Bachheti, "Differential pulse voltammetric determination of paracetamol at nanogold modified indium tin oxide electrode," Electrochemistry Communications, vol. 7, no. 8, pp. 803-807, 2005.

[12] B. C. Lourencao, R. A. Medeiros, R. C. Rocha-Filho, L. H. Mazo, and O. Fatibello-Filho, "Simultaneous voltammetric determination of paracetamol and caffeine in pharmaceutical formulations using a boron-doped diamond electrode," Talanta, vol. 78, no. 3, pp. 748-752, 2009.

[13] N. Spataru, B. V. Sarada, D. A. Tryk, and A. Fujishima, "Anodic voltammetry of xanthine, theophylline, theobromine and caffeine at conductive diamond electrodes and its analytical application," Electroanalysis, vol. 14, no. 11, pp. 721-728, 2002.

[14] M. Z. Ding and J. K. Zou, "Centrifugal extraction procedure for the ultraviolet spectrophotometric determination of caffeine in beverages," Chinese Journal of Analytical Chemistry, vol. 36, no. 3, pp. 381-384, 2008.

[15] Y. Yamauchi and A. NakamuraI. Kohno, M. Kitai, K. Hatanaka, and T. Tanimoto, "Simple and rapid UV spectrophotometry of caffeine in tea coupled with sample pre-treatment using a cartridge column filled with polyvinylpolypyrrolidone (PVPP)," Chemical \& Pharmaceutical Bulletin, vol. 56, no. 2, pp. 185-188, 2008.

[16] Y. Yamauchi and A. NakamuraI. Kohno, K. Hatanaka, M. Kitai, and T. Tamimoto, "Quasi-flow injection analysis for rapid determination of caffeine in tea using the sample pre-treatment method with a cartridge column filled with polyvinylpolypyrrolidone," Journal of Chromatography. A, vol. 1177, no. 4, pp. 190-194, 2008.

[17] A. Espinosa-Mansilla, F. Salinas, and I. De Orbe Paya, "Simultaneous determination of sulfadiazine, doxycycline, furaltadone and trimethoprim by partial last squares multivariate calibration," Analytica Chimica Acta, vol. 313, no. 12, pp. 103-112, 1995.

[18] P. Hubert, J. J. Nguyen-Huu, B. Boulanger et al., "Harmonization of strategies for the validation of quantitative analytical procedures: a SFSTP proposal-part II," Journal of Pharmaceutical and Biomedical Analysis, vol. 45, no. 1, pp. 70-81, 2007.

[19] P. Hubert, J. J. Nguyen-Huu, B. Boulanger et al., "Harmonization of strategies for the validation of quantitative analytical procedures: a SFSTP proposal-part I," Journal of Pharmaceutical and Biomedical Analysis, vol. 36, no. 3, pp. 579-586, 2004.

[20] Z. Xiaobo, Z. Jiewen, M. J. W. Povey, M. Holmes, and M. Hanpin, "Variables selection methods in near-infrared spectroscopy," Analytica Chimica Acta, vol. 667, no. 1-2, pp. 14-32, 2010.

[21] J. L. Ramirez, M. K. Bellamy, and R. J. Romañach, "A novel method for analyzing thick tablets by near infrared spectroscopy," AAPS PharmSciTech, vol. 2, no. 3, pp. 15-24, 2001.

[22] I. Tomuta, L. Rus, R. Iovanov, and L. L. Rus, "High-throughput NIR-chemometric methods for determination of drug content and pharmaceutical properties of indapamide tablets," Journal 
of Pharmaceutical and Biomedical Analysis, vol. 84, pp. 285292, 2013.

[23] I. Tomuta, R. Iovanov, E. Bodoki, and L. Vonica, "Development and validation of NIR-chemometric methods for chemical and pharmaceutical characterization of meloxicam tablets," Drug Development and Industrial Pharmacy, vol. 40, no. 4, pp. 549-559, 2014.

[24] C. P. Meza, M. A. Santos, and R. J. Romanach, "Quantitation of drug content in a low dosage formulation by transmission near infrared spectroscopy," AAPS PharmSciTech, vol. 7, no. 1, article 29, pp. E1-E9, 2006.

[25] A. Porfire, I. Tomuta, L. Tefas, S. E. Leucuta, and M. Achim, "Simultaneous quantification of $1-\alpha$-phosphatidylcoline and cholesterol in liposomes using near infrared spectrometry and chemometrics," Journal of Pharmaceutical and Biomedical Analysis, vol. 63, pp. 87-94, 2012.

[26] A. Porfire, D. Muntean, M. Achim, L. Vlase, and I. Tomuta, "Simultaneous quantification of simvastatin and excipients inliposomes using near infrared spectroscopy and chemometry," Journal of Pharmaceutical and Biomedical Analysis, vol. 107, pp. 40-49, 2015. 

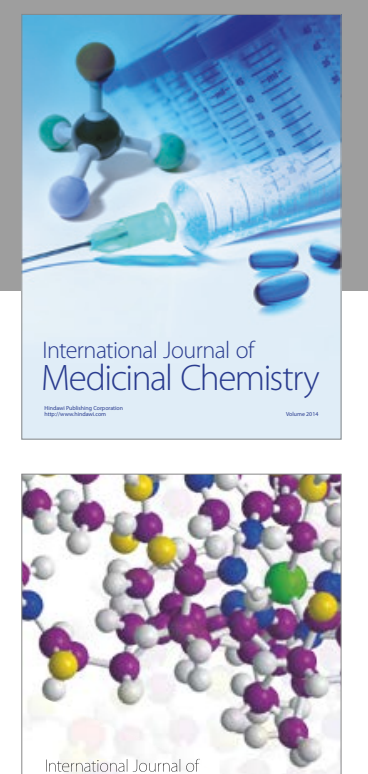

Carbohydrate Chemistry

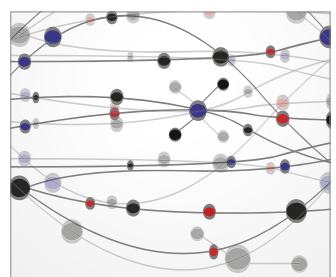

The Scientific World Journal
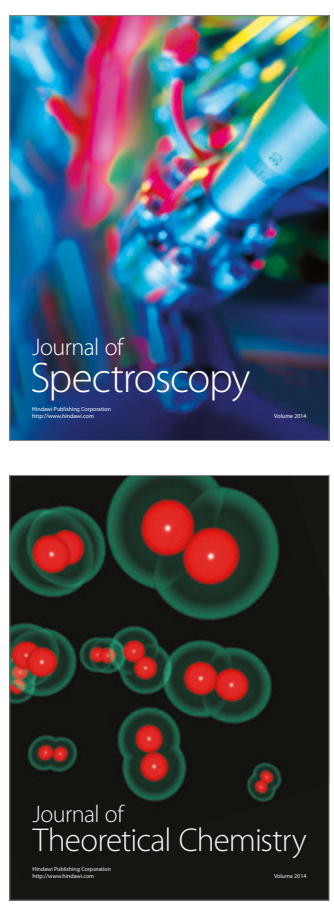
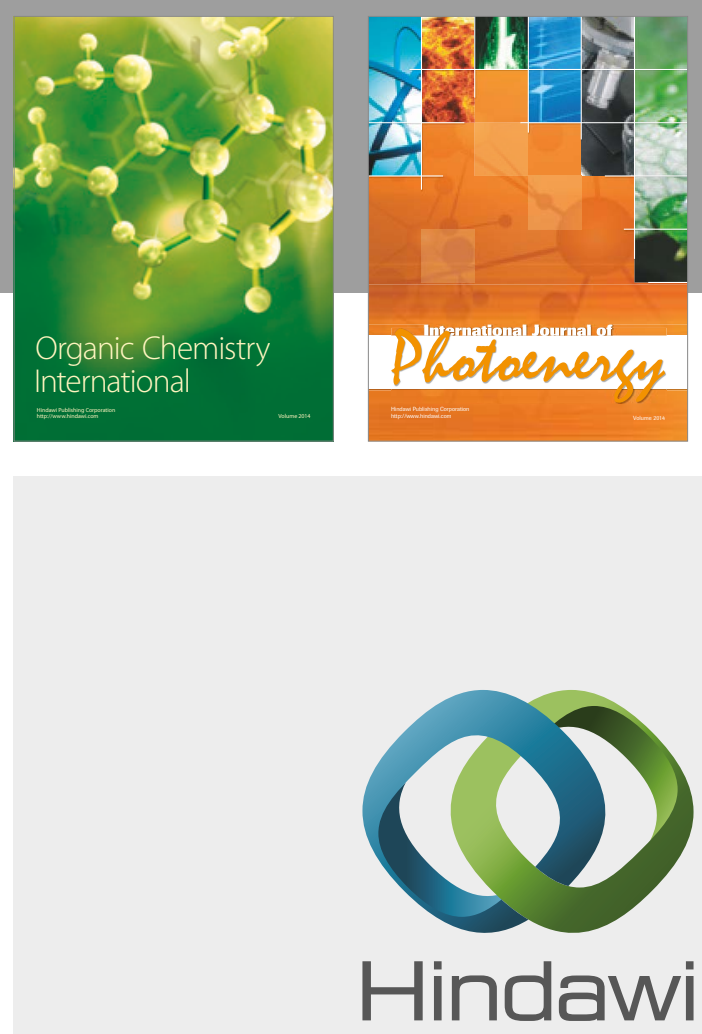

Submit your manuscripts at

https://www.hindawi.com

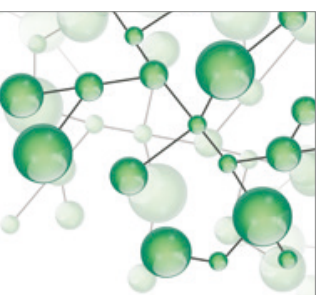

International Journal of

Inorganic Chemistry

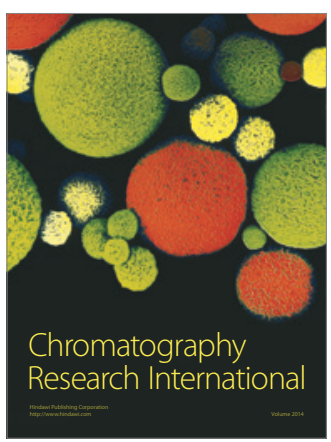

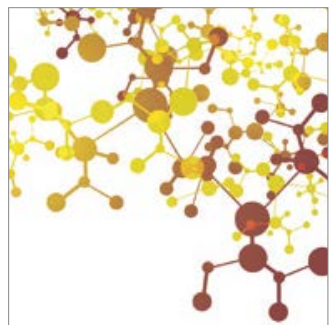

Applied Chemistry
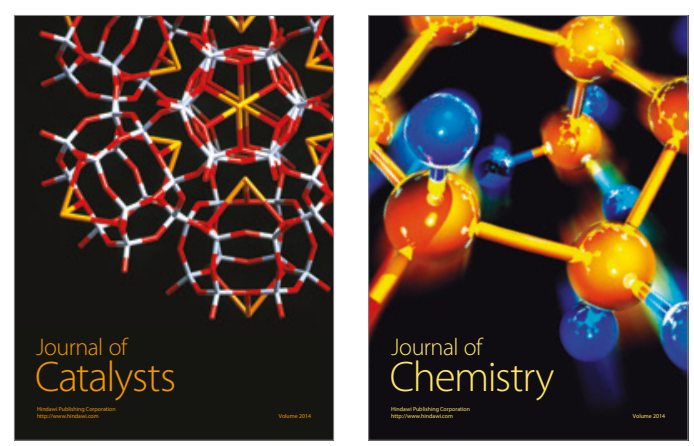
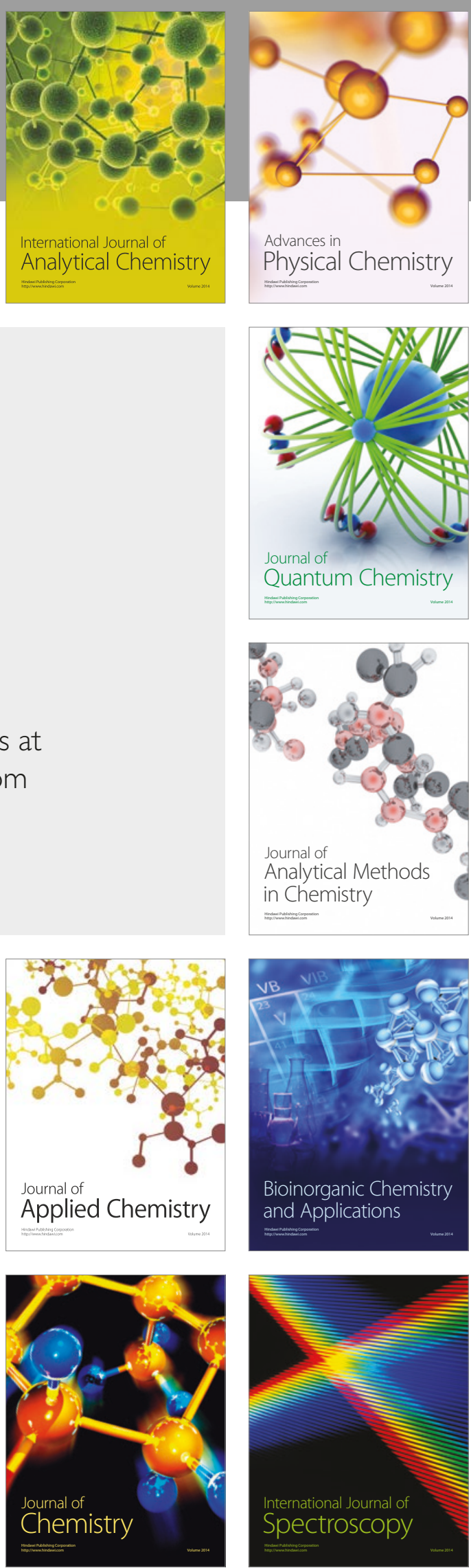